\title{
GENERALIZED UNKNOTTING OPERATIONS AND TANGLE DECOMPOSITIONS
}

\author{
TSUYOSHI KOBAYASHI
}

(Communicated by Haynes R. Miller)

\begin{abstract}
Suppose that a knot $K_{L}$ in $S^{3}$ is obtained from a knot $K$ by an $n$-parallel ( $n$-antiparallel resp.) crossing change. Let $T$ be an incompressible, $\partial$-incompressible surface properly embedded in $S^{3}-\dot{N}(K)$ with $\partial T$ a union of meridian loops and $\chi(T)>p(1-2 n)$, for some $p$. We show that either $T$ is isotoped to intersect $L$ in $\leq 2 p-2$ points, or there is a minimal genus Seifert surface for $K_{L}$ intersecting the corresponding crossing link in two $(\leq 2$ resp.) points.
\end{abstract}

\section{INTRODUCTION}

Let $K$ be a knot in the 3-sphere $S^{3}$ and $D$ a disk which intersects $K$ transversely in two points. Then let $L=\partial D$. Fix a natural number $n$. We get a knot $K_{L}$ in $S^{3}$ as the image of $K$ after doing $\pm 1 / n$ surgery along $L$. We say that $K_{L}$ is obtained from $K$ by a single $n$-parallel crossing change or $n$-antiparallel crossing change if $K$ intersects $D$ in two points with the same orientation or different orientations respectively. Then $D$ is called a crossing disk and $L$ is a crossing link. Let $L^{\prime}$ be the crossing link for $K_{L}$ corresponding to $L$. In [S-T, §3], Scharlemann-Thompson studied some relations between the genera $g(K), g\left(K_{L}\right)$ of $K, K_{L}$ and incompressible tori in $S^{3}-\dot{N}(K)$ in case of $n=1$. In fact, they proved:

Theorem [S-T, Theorem 3.1, Corollary 3.2]. Suppose $K$ and $K_{L}$ are related by a single 1-crossing change. Let $T$ be an incompresssible torus in $S^{3}-\dot{N}(K)$. The either $T$ is isotoped to be disjoint from $L$ in $S^{3}-\dot{N}(K)$ or there is a minimal genus Seifert surface for $K_{L}$ intersecting $L^{\prime}$ in $\leq 2$ points. Moreover if $g\left(K_{L}\right) \leq g(K)-2$ then $T$ can be isotoped to be disjoint from $L$.

In this paper we generalize the result as follows.

Received by the editors March 30, 1987 and, in revised form, October 20, 1987.

1980 Mathematics Subject Classification (1985 Revision). Primary 57M25; Secondary 57N10.

Key words and phrases. Knot, unknotting operation, tangle, sutured manifold.

This work was supported by Grant-in-Aid for Scientific Research No. 61460003, The Ministry of Education of Japan. 
Theorem 1. Suppose that $K_{L}$ is obtained form $K$ by a single $n$-parallel crossing change. Let $T$ be an incompressible, $\partial$-incompressible (possibly closed) surface properly embedded in $S^{3}-\dot{N}(K)$ such that $\partial T$ is a union of meridian loops and $\chi(T)>p(1-2 n)$, for some $p$. Then either $T$ is properly isotopic to a surface intersecting $L$ in $\leq 2 p-2$ points, or there is a minimal genus Seifert surface for $K_{L}$ intersecting $L^{\prime}$ in two points.

In case of antiparallel crossing change we have a result a little bit stronger.

Theorem 2. Suppose that $K_{L}$ is obtained form $K$ by a single $n$-antiparallel crossing change. Let $T, p$ be as in Theorem 1. Then either $T$ is properly isotopic to a surface intersecting $L$ in $\leq 2 p-2$ points, or there is a minimal genus Seifert surface for $K_{L}$ intersecting $L^{\prime}$ in $\leq 2$ points. Moreover if $\chi(T)>$ $p(2-2 n)$ then we can take the minimal genus Seifert surface to be disjoint from $L^{\prime}$.

As consequences of Theorems 1, 2 we have

Corollary 1. Let $K, L, K_{L}, T$ be as in Theorem 1. If $g\left(K_{L}\right) \leq g(K)-(n+1)$ then $T$ is properly isotopic to a surface intersecting $L$ in $\leq 2 p-2$ points.

Corollary 2. Let $K, L, K_{L}, T$ be as in Theorem 2. If either $g\left(K_{L}\right) \leq g(K)-2$ or $\chi(T)>p(2-2 n)$ and $g\left(K_{L}\right) \leq g(K)-1$ then $T$ is properly isotopic to a surface intersecting $L$ in $\leq 2 p-2$ points.

A ( $m$-string) tangle is a pair $(B, t)$ where $B$ is a 3-cell and $t$ is a union of $m$ mutually disjoint arcs properly embedded in $B$. A tangle $(B, t)$ is inseparable if $t$ cannot be separated by a disk properly embedded in $B$. We note that typical examples of $T$ in Theorems 1,2 are planar surfaces which give tangle decompositions of $K$ into inseparable tangles in case of $m>1$ or into nontrivial tangles in case of $m=1$. Hence we have:

Corollary 3. Let $K, L, K_{L}$ be as in Theorem 1. Suppose that $K$ has a tangle decomposition $K=\left(B_{1}, t_{1}\right) \cup\left(B_{2}, t_{2}\right)$, where $\left(B_{i}, t_{i}\right) \quad(i=1,2)$ is an $m(\leq n)$ string tangle with either $\left(B_{i}, t_{i}\right) \quad(i=1,2)$ inseparable or $m=1$ and $\left(B_{i}, t_{i}\right)$ $(i=1,2)$ nontrivial. Suppose that $2-2 m>p(1-2 n)$ for some $p>0$. Then either $L$ is ambient isotopic in $S^{3}-K$ to a knot intersecting $\partial B_{i}$ in $\leq 2 p-2$ points, or there is a minimal genus Seifert surface for $K_{L}$ intersecting $L^{\prime}$ in two points. Moreover if $g\left(K_{L}\right) \leq g(K)-(n+1)$, then $L$ is ambient isotopic in $S^{3}-K$ to a knot intersecting $\partial B_{i}$ in $\leq 2 p-2$ points.

Corollary 4. Let $K, L, K_{L}$ be as in Theorem 2. Suppose that $K$ has a tangle decomposition as in Corollary 3 and that $2-2 m>p(1-2 n)$, for some $p>0$. Then either $\downarrow$ is ambient isotopic in $S^{3}-K$ to a knot intersecting $\partial B_{i}$ in $\leq 2 p-2$ points, or there is a minimal genus Seifert surface for $K_{L}$ intersecting $L^{\prime}$ in $\leq 2$ points. Moreover if $g\left(K_{L}\right) \leq g(K)-2$ or $g\left(K_{L}\right) \leq g(K)-1$ and $2-2 m>p(2-2 n)$, then $L$ is ambient isotopic in $S^{3}-K$ to a knot intersecting $\partial B_{i}$ in $\leq 2 p-2$ points. 

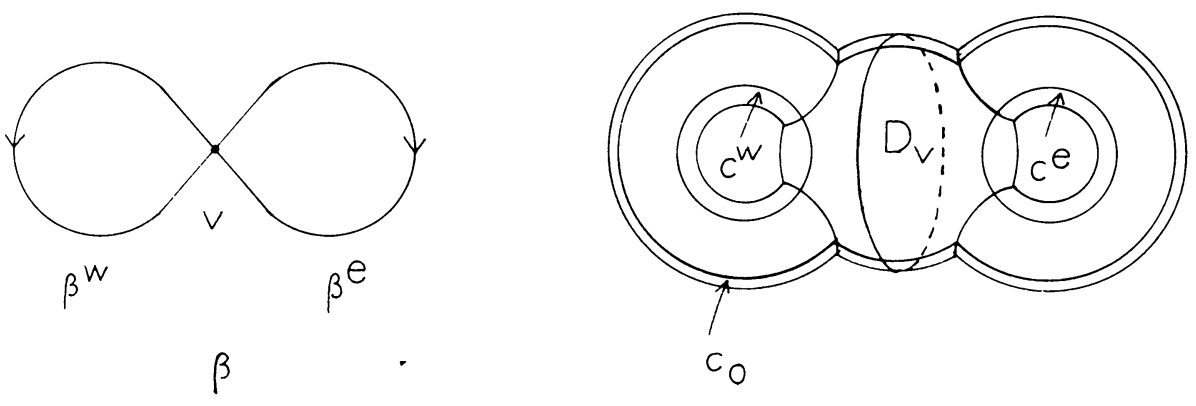

FIGURE 2.1

Throughout this paper, we will work in the piecewise linear category. The proofs heavily depend on the results and the arguments in [S, S-T]. We assume that the reader is familiar with the papers.

For the notations of $\mu, \nu, K$ see [S]. For the notations $\lambda^{e}, \lambda^{w}, \lambda^{n}, \lambda^{s}, \lambda^{\prime}, \lambda^{\prime}$ and the definition of $\kappa$ see [S-T, §2].

\section{THEOREM 1}

In this section we prove Theorem 1. The proof is carried out by using the same machine as in [S-T]. Let $\beta \subset \mathbf{R}^{3}$ be a 1-complex each edge of which has an orientation and $W$ a regular neighborhood of $\beta$ as in Figure 2.1. Let $D_{v}$ be a disk properly embedded in $W, c_{0}, c^{w}, c^{e}$ simple closed curves on $\partial W$ as in Figure 2.1. Let $\bar{c}$ be the simple closed curve obtained from $c_{0}$ by doing a half twist along $\partial D_{v}$. Then let $c^{0}$ be the simple closed curve obtained from $\partial D_{v}$ be doing Dehn twists $n$-times along $\bar{c}$. Suppose that $\beta$ is embedded in a compact orientable 3-manifold $M$ containing no lens space summand and $\partial M$ a torus, where we regard the regular neighborhood $N(\beta)$ of $\beta$ as $W$. Suppose that $M$ is $\beta$-irreducible [S] and that there is an incompressible, $\partial$-incompressible surface $(Q, \partial Q) \subset(M-\dot{W}, \partial W \cup \partial M)$ consisting of three components $Q^{e}, Q^{w}$ and $Q^{0}$ with the following properties:

(a) $Q^{w}$ is an annulus with boundary the union of $c^{w}$ and a simple closed curve $\mu \subset \partial M$ which is essential in $M$. We suppose that $\mu$ has the orientation induced from $\beta^{w}$,

(b) Similarly $Q^{e}$ is an annulus with boundary the union of $c^{e}$ and a simple closed curve $\mu^{\prime}$. If $\mu^{\prime}$ has the orientation induced form $\beta^{e}$ then $\mu^{\prime}$ and $\mu$ are parallel oriented in $\partial M$,

(c) $Q^{0}$ is an incompressible, $\partial$-incompressible surface such that $\partial Q^{0}$ consists of $p(\geq 1)$ copies of $c^{0}$ in $\partial W$ and (possibly empty) collection of essential loops of $\partial M$ with $\chi\left(Q^{0}\right)+p>p(1-2 n)$.

We note that $c^{0}$ contains one $\lambda^{n}$ and $\lambda^{s}$, and $2 n-1 \lambda^{\prime}$ 's and $\lambda^{\prime}$ 's, and has $\nu=0, \mu=4 n, K=-2-2+(2 n-1)(-1-1)=-4 n-2$. Hence the index [S] of $Q^{0}$ is $I\left(Q^{0}\right)=p(4 n-4 n-2)-2 \chi\left(Q^{0}\right)=-2\left(p+\chi\left(Q^{0}\right)\right)<2 p(2 n-1)$. 
Then we have

Lemma 2.1. If $(S, \partial S) \subset(M, \partial M)$ is $\beta$-taut and $\partial S \neq \varnothing$ then $S$ is taut in the Thurston norm.

Proof. By [S], there is a $\beta$-taut sutured manifold hierarchy

$$
(M, \partial M, \beta) \stackrel{S_{1}=S}{\longrightarrow}\left(M_{1}, \gamma_{1}, \beta_{1}\right) \stackrel{S_{2}}{\longrightarrow} \cdots \stackrel{S_{m}}{\longrightarrow}\left(M_{m}, \gamma_{m}, \beta_{m}\right)
$$

respecting $Q$. Then the proof of Lemma 2.1 is done by using the argument of the proof of [S-T, Lemma 2.2]. We note that almost all the proofs there work in this situation. Actually the major claim (a) and claims 1-8 are valid without changing proofs. To sum up the results we have:

FACT 0 . There is a cancelling disk for every arc component of every $\beta_{i}$;

FACT 1. Each edge $\beta^{w}$ and $\beta^{e}$ intersects nontrivially one of the decomposing surfaces $S_{i}$;

FACT 2. Each component $q$ of $Q_{i}^{w}$ for which $\partial q \cap \dot{R}\left(\beta_{i}^{w}\right) \neq \varnothing$ is a disk such that $\partial q \cap \beta_{i}^{w}$ is a single arc and $\partial q \cap \partial M_{i}$ is a single arc crossing a single suture.

To state Facts 3-5, we prepare some notations. Fact 0 together with [S, 4.16(c)] shows that every $S_{i}$ is conditioned [S, 2.4]. Hence, by [S, 3.3, 3.9], we see that if $\left(M_{m}, \gamma_{m}\right)$ is taut in the Thurston norm, then $S$ is taut in the Thurston norm. $\beta$ denotes the component of $\beta_{m}$ containing the vertex $v$. Fact 1 shows that $\beta$ is $X$-shaped. Let $q^{w}$ ( $q^{e}$ resp.) be the component of $Q_{m}^{w}\left(Q_{m}^{e}\right.$ resp.) which satisfies the conclusion of Fact 2. $\beta \cap \partial M_{m}$ consists of four points $\varepsilon^{n w}, \varepsilon^{n e}, \varepsilon^{s w}, \varepsilon^{s e}$ as in Figure 2.2. Then we have:

FACT 3. Each disk component of $R_{ \pm}$intersects $\beta$, where $R_{ \pm}=R_{ \pm}\left(\gamma_{m}\right)$.

FACT 4. If $\varepsilon^{n w}$ and $\varepsilon^{n e}$ (or $\varepsilon^{s w}$ and $\varepsilon^{s e}$ ) lie in the same component of $R_{ \pm}$ then $\left(M_{m}, \gamma_{m}\right)$ is taut in the Thurston norm.

FACT 5. If any component of $Q_{m}^{0}$ has negative index then $\left(M_{m}, \gamma_{m}\right)$ is taut in the Thurston norm.

Assume that $\left(M_{m}, \gamma_{m}\right)$ is not taut in the Thurston norm. Then, by Facts 2,3 and 4 , if necessary by exchanging $\varepsilon^{n w}, \varepsilon^{s w}$ and $\varepsilon^{n e}, \varepsilon^{s e}$, we see that the suture of the component of $\left(M_{m}, \gamma_{m}\right)$ containing $\beta$ is as in Figure 2.2.

Claim. Let $l$ be a component of $\partial Q_{m}^{0}$. Suppose that $\ln \beta$ contains $r(\geq 1)$ arcs running from $\varepsilon^{n e}$ to $\varepsilon^{s w}$. Then $l$ has $\mu+\nu+K \geq 4 r$.

Proof. Let $A_{1}, \ldots, A_{r}$ be the subarcs of $l$ running from $\varepsilon^{n e}$ to $\varepsilon^{\text {sw }}$. Since each $A_{i}$ contributes 1 to $\mu+\nu+K$, it is enough to show that each component of $l-\left(A_{1} \cup \cdots \cup A_{r}\right)$ contributes 3 to $\mu+\nu+K$. A case by case analysis as in Figure 2.3 shows that this is true.

Conclusion of proof. Let $\bar{Q}$ be the union of the components of $Q_{m}^{0}$ whose boundary contains an arc on $\beta$ running from $\varepsilon^{n e}$ to $\varepsilon^{s w}$. Since $\partial S \neq \varnothing$ we have the condition (c) $\partial Q_{m}^{0} \cap \beta$ contains $p(2 n-1)$ arcs running from $\varepsilon^{n e}$ 


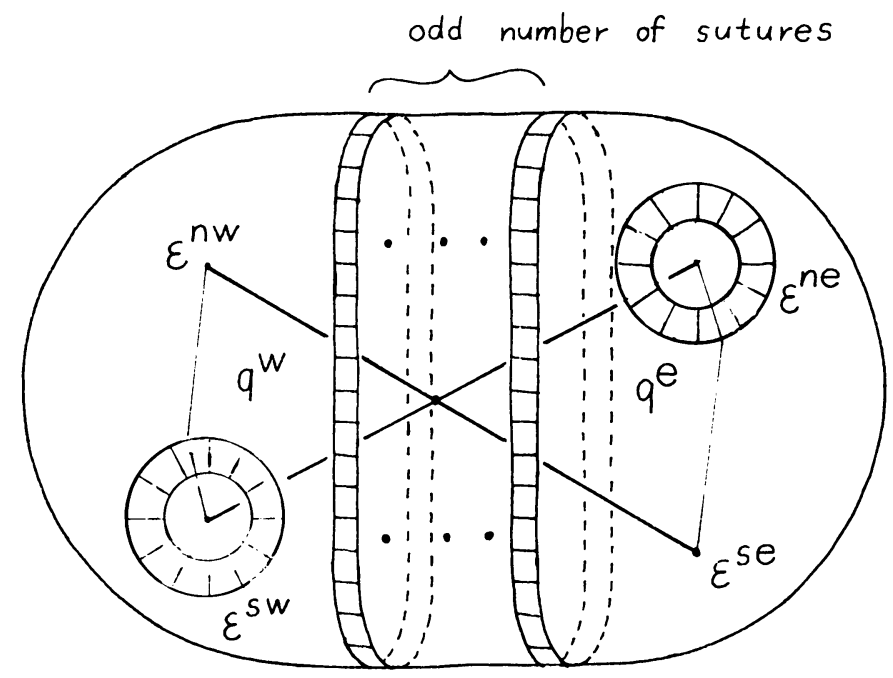

FIGURE 2.2
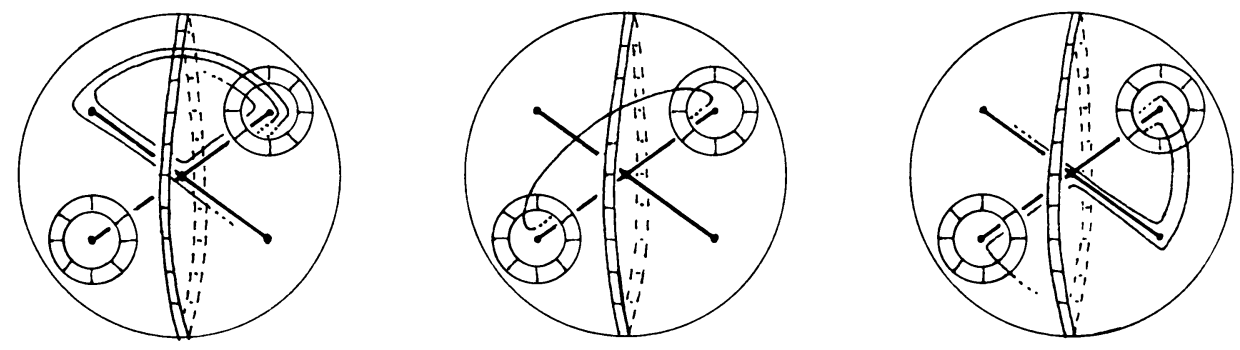

FIGURE 2.3

to $\varepsilon^{s w}$. Hence, we see, by claim, that $\bar{Q}$ contributes at least $4 p(2 n-1)$ to $\mu+\nu+K$. Since $\bar{Q}$ contains at most $p(2 n-1)$ components we have $I(\bar{Q}) \geq$ $4 p(2 n-1)-2 p(2 n-1)>I\left(Q^{0}\right)$. Since $I\left(Q_{m}^{0}\right) \leq I\left(Q^{0}\right)$, some component of $Q_{m}^{0}$ has negative index, contradicting Fact 5 .

This completes the proof of Lemma 2.1.

Proof of Theorem 1. Suppose that the number of the components of $T \cap D$ is minimal among all surfaces properly isotopic to $T$. Suppose that $L$ intersects $T$ in $>2 p-2$ points. Let $\beta\left(\subset S^{3}-\dot{N}\left(K_{L}\right)\right)$ be as in Figure 2.4. Then we can get a surface satisfying the assumption of Lemma 2.1 from $T$. For the detailed argument, see the proof of [S-T, Theorem 3.1]. We note that $\beta$-taut Seifert surface for $K_{L}$ intersects $\beta$ in two points. Hence, by Lemma 2.1, there is a minimal genus Seifert surface $S$ for $K_{L}$ intersecting $L^{\prime}$ in two points.

Proof of Corollaries 1, 3. Let $K, K_{L}, T$ be as in Corollary 1. Assume that $T$ can not be isotoped to intersect $L$ in $\leq 2 p-2$ points. Then, by Theorem 1 , there is a minimal genus Seifert surface $S$ for $K_{L}$ intersecting $L^{\prime}$ in two 

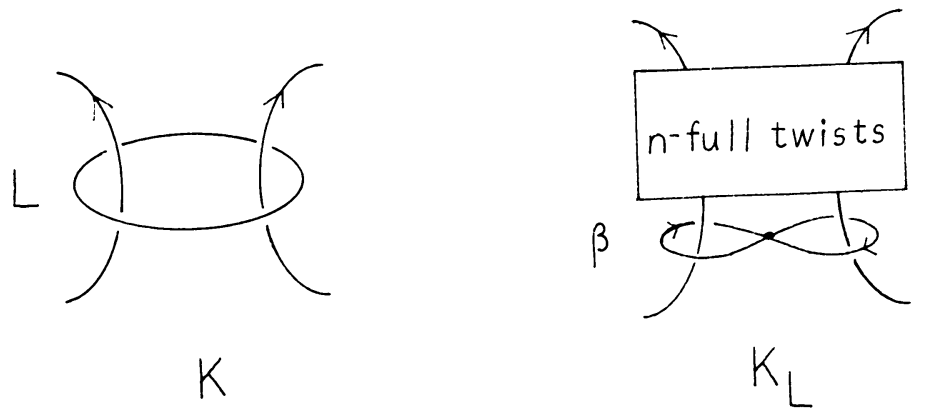

FIGURE 2.4
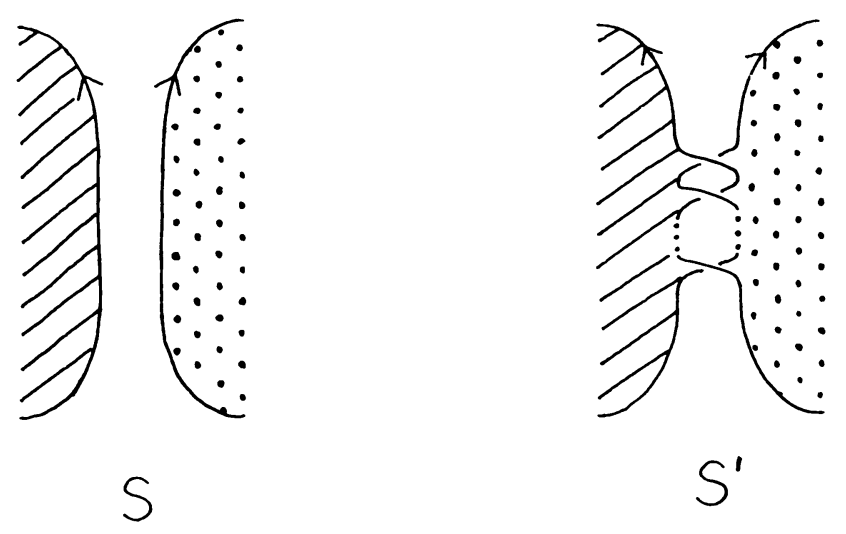

FIGURE 2.5

points. We can attach $2 n$ half twisting bands to $S$ in a neighborhood of a disk bounded by $L^{\prime}$ to have a Seifert surface $S^{\prime}$ for $K$ such that genus $\left(S^{\prime}\right)=$ genus $(S)+n$. See Figure 2.5. Hence $g(K) \leq g\left(K_{L}\right)+n$, a contradiction. Corollary 3 is an immediate consequence of Theorem 1 and Corollary 1 .

\section{THEOREM 2}

In this section we prove Theorem 2. Let $\beta, c^{w}, c^{e}, c_{0}, W, D_{v}$ be as in $\S 2$. Let $c^{0}$ be the simple closed curve in $\partial W$ obtained from $\partial D_{v}$ by doing Dehn twist $n$ times along $c_{0}$. Suppose that $\beta$ is embedded in a compact 3-manifold $M$ containing no lens space summand and $\partial M$ a torus, where we regard $N(\beta)$ as $W$. Suppose that $M$ is $\beta$-irreducible and there is an incompressible, $\partial$ incompressible surface $(Q, \partial Q) \subset(M-\dot{W}, \partial W \cup \partial M)$ consisting of three components $Q^{e}, Q^{w}$ and $Q^{0}$ with the same properties (a), (b), (c) as in $\S 2$. Then we have

Lemma 3.1. If $(S, \partial S) \subset(M, \partial M)$ is $\beta$-taut then $S$ is taut in the Thurston norm. 
Proof. The proof is essentially the same as that of Lemma 2.1. Hence we just sketch how the proof proceeds. Let $\left(M_{m}, \gamma_{m}, \beta_{m}\right), Q_{m}^{0}$ be as in the proof of Lemma 2.1. We note that Facts $0-5$ holds in this situation. $c^{0}$ has $\nu=0$, $\mu=4 n$ and $K=-8 n+2$ so that $I\left(Q^{0}\right)=(4 n-8 n+2) p-2 \chi\left(Q^{0}\right)<$ $-4 n p+2 p+4 n p=2 p$. On the other hand $\partial Q_{m}^{0} \cap \beta$ contains $p$ arcs running from $\varepsilon^{n e}$ to $\varepsilon^{s w}$. Hence, if $\left(M_{m}, \gamma_{m}\right)$ is not taut in the Thurston norm, then, by Claim, some component of $Q_{m}^{0}$ has negative index, contradicting Fact 5 . Hence $\left(M_{m}, \gamma_{m}\right)$ is taut in the Thurston norm so that $S$ is taut in the Thurston norm.

Then the proof of the first half of the conclusion of Theorem 2 is carried out by using Lemma 3.1 and the arguments of the proof of Theorem 1. Hence, in the rest of this section, we prove the latter half of Theorem 2, i.e. the case of $\chi(T)>p(2-2 n)$.

Let $K, K_{L}$ be as in Theorem 2. Let $D$ be the crossing disk bounded by $L$. Suppose that $T$ cannot be isotoped to intersect $L$ in $\leq 2 p-2$ points. Then we get $\beta \subset S^{3}-\dot{N}\left(K_{L}\right)$ and $Q\left(\subset M_{\beta}=S^{3}-\left(\dot{N}\left(K_{L}\right) \cup \dot{N}(\beta)\right)\right)$ which satisfies the assumption of Lemma 3.1, by using the argument as in [S-T, Theorem 3.1]. Let $S$ be a minimal genus Seifert surface for $K_{L}$ intersecting $L^{\prime}$ in $\leq 2$ points. Suppose that $S$ intersects $L^{\prime}$ in two points. Let $S^{0}=S-\dot{N}(\beta)$. Each component of $\partial Q^{0}$ contained in $\partial W$ is divided by $S^{0}$ into $4 n$ arcs, one of which is of the form $\lambda^{\prime}$ and another of the form $\lambda \backslash$. These two arcs are called "bad", so of the $4 p n$ arcs of $\left(\partial Q^{0} \cap \partial W\right)-S^{0}, 2 p$ are bad. Since $\partial T$ is a union of meridians, $\partial T$ consists of even number, say $2 q$, of components. Then the $2 q$ boundary components of $\partial Q^{0}$ corresponding to $\partial T$ become arcs in $\partial Q^{0}-S^{0}$, which we also call "bad". The other $2 p(2 n-1)$ "good" arcs in $\partial Q^{0}-S^{0}$ are of the form $\lambda^{n}$ or $\lambda^{s}$.

Since $M$ is $\beta$-irreducible we may suppose that every simple closed curve in $Q^{0} \cap S^{0}$ is essential in both $Q^{0}$ and $S^{0}$. Let $Q^{-}$denote $Q^{0}-S^{0}$, and $\left\{q_{\rho}\right\}$ the set of components of $Q^{-}$. Since $Q^{0} \cap S^{0}$ consists of $(4 p n+2 q) / 2=2 p n+q$ arcs and some simple closed curves, $\chi\left(Q^{-}\right)=\sum_{\rho} \chi\left(q_{\rho}\right)=\chi\left(Q^{0}\right)+2 p n+q$.

Claim 1. Some component of $Q^{-}$is a disk whose boundary contains no bad arc.

Proof. Any component of $\partial Q^{-}$contains an even number of bad arcs, for bad arcs are precisely those with one end on each side of $S^{0}$. Hence there are at most $p+q$ disks whose boundary contains bad arcs. Since $\chi\left(Q^{0}\right)>p(2-2 n)-p$, we have $\chi\left(Q^{-}\right)>(p(2-2 n)-p)+(2 p n+q)=p+q$. Since only disk components of $Q^{-}$have positive Euler characteristic, the claim follows.

Let $q^{-}$be a component of $Q^{-}$whose boundary contains no bad arc. Since $\partial q^{-}$is not a closed curve of $S^{0} \cap Q^{0}, \partial q^{-}$contains a good arc of the form, say, $\lambda^{n}$. By tubing together the points of intersection of $L^{\prime}$ and $S$ in the north side of $S$, we get a new Seifert surface $S^{\prime \prime}$ for $K_{L}$ which is disjoint form $L^{\prime}$, but of one higher genus than $S$. 
Claim 2. $\partial q$ is essential in $S^{\prime \prime}$.

Proof. Suppose not, so $\partial q$ bounds a disk $E$ in $S^{\prime \prime}$. Let $\delta$ denote a meridian of the tube $S^{\prime \prime}-S^{0}$, and consider an outermost arc of $\delta \cap E$ in $E$. It cuts off from $E$ a $\partial$-compressing disk for $Q^{0}$, a contradiction.

By doing surgery on $S^{\prime \prime}$ along $\partial q$ we obtain a Seifert surface $S^{\prime}$ for $K_{L}$ such that $S^{\prime} \cap L^{\prime}=\varnothing$ and genus $\left(S^{\prime}\right)=$ genus $(S)$.

This completes the proof of Theorem 2 .

Corollaries 2,4 are proved by Theorem 2 and the arguments of the proof of [S-T, Corollary 3.2]. Hence we omit the proofs.

\section{ACKNOWLEDGMENT}

I would like to express my thanks to the referee for pointing out a defect of proof in the manuscripts, and many useful suggestions.

\section{REFERENCES}

[S] M. Scharlemann, Sutured manifolds and generalized Thurston norm, preprint.

[S-T] M. Scharlemann and A. Thompson, Unknotting number, genus, and companion tori, preprint.

Department of Mathematics, Osaka University, Toyonaka, Osaka 560, Japan 